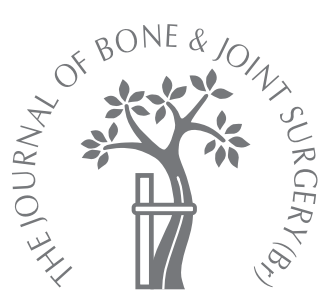

S. Church,

J. F. Keating

From The Royal

Infirmary of

Edinburgh, Scotland

S. Church, MB ChB, House

Officer

J. F. Keating, FRCS Ed

(Orth), Consultant

Orthopaedic Surgeon

Department of Orthopaedic

Trauma

Royal Infirmary, Little France,

Old Dalkeith Road,

Edinburgh EH16 4SU, UK.

Correspondence should be sent to Mr J. F. Keating:

e-mail:

john.keating@ed.ac.uk

(C)2005 British Editorial

Society of Bone and

Joint Surgery

doi:10.1302/0301-620X.87B12.

$16916 \$ 2.00$

$J$ Bone Joint Surg [Br] 2005;87-B:1639-42.

Received 1 July 2005;

Accepted 19 July 2005

\section{Reconstruction of the anterior cruciate ligament}

\author{
TIMING OF SURGERY AND THE INCIDENCE OF MENISCAL TEARS \\ AND DEGENERATIVE CHANGE
}

We reviewed 183 patients who had undergone reconstruction of the anterior cruciate ligament. The incidence of meniscal tears and degenerative change was assessed and related to the timing from injury to surgery. Degenerative change was scored using the French Society of Arthroscopy system. The patients were divided into an early (surgery within 12 months of injury) and a late group (surgery more than 12 months from injury). The late group was also subdivided into four groups of 12-month periods ranging from one year to more than four years after injury.

There was a significantly higher incidence of meniscal tears in patients undergoing reconstruction after 12 months compared with those in the early group $(71.2 \%$ vs $41.7 \%$; p $<0.001$ ). This was due to a large increase in medial meniscal tears in the late group. An increased incidence of degenerative change was also found in the late group $131.3 \%$ vs $10.7 \% ; p<0.001$ ). Analysis of the subgroups showed that the incidence of meniscal tears and degenerative change did not differ significantly when surgery was performed after 12 months from injury. We conclude that reconstruction of the anterior cruciate ligament should be carried out within 12 months of injury to minimise the risk of meniscal tears and degenerative change.

The incidence of meniscal tears is increased in the anterior cruciate ligament (ACL)-deficient knee and there is some evidence to indicate that degenerative changes develop with time. It is known that reconstruction of the ACL reduces the incidence of meniscal tears. The relationship of reconstruction to the prevention of osteoarthritis is more controversial. ${ }^{1}$ There are a number of outcome studies which have examined the long-term results of patients who have had reconstruction of the ACL. ${ }^{2-5}$ These and other studies have made observations in regard to meniscal tears in both the ACL-deficient and reconstructed knee. ${ }^{6-9}$ In general, early rather than late reconstruction has been recommended to minimise the risk of meniscal tears. Some studies have compared surgical with conservative treatment and have shown an improved outcome in the surgical groups even when surgery was performed late after injury. ${ }^{10,11}$ One study showed that delayed reconstruction in the young was associated with a higher incidence of medial meniscal tears. ${ }^{12}$ Another examined the different patterns of meniscal tear in acute, subacute, subchronic and chronic ACL ruptures and showed an increased incidence of medial meniscal tears in chronic ACL-deficient knees. ${ }^{13}$
However, there are no clear guidelines regarding the timing of reconstruction in regard to reducing the rate of meniscal pathology, and timing remains controversial. There has been an increasing trend towards earlier reconstruction on the basis that the functional outcome is better and that it minimises the risk of meniscal pathology. In a previous randomised study, we have shown that there is no functional advantage of immediate over delayed reconstruction at three months after injury. ${ }^{14}$ It is unclear, however, as to how long reconstruction can be delayed before meniscal tears and degenerative change begin to occur. In this study we recorded the incidence of meniscal tears and degenerative change at the time of reconstruction of the ACL and related the findings to the time elapsed since injury. Our aim was to determine the safe period in which to perform reconstruction before the development of meniscal tears and degenerative change in the ACL-deficient knee.

\section{Patients and Methods}

We reviewed 183 patients younger than 40 years of age who had undergone reconstruction of the ACL between 1996 and 2002 at the Edinburgh Royal Infirmary. There were 135 
Table I. Age and gender distribution across subgroups $A$ to $E$

\begin{tabular}{lllllll}
\hline & \multicolumn{1}{l}{ Subgroup $^{*}$} & & & \\
\cline { 2 - 7 } & A (0 to 12) & B (13 to 24) & C (25 to 36) & D (37 to 48) & E (> 48) \\
\hline Male:Female & $78: 25$ & $18: 4$ & $12: 6$ & $9: 4$ & $18: 9$ & $29.4(30)$ \\
Mean age in yrs (median) & $25.5(25)$ & $26.7(27)$ & $28.5(28.5)$ & $26.9(28)$ & 29.4 \\
\hline
\end{tabular}

* time in months after surgery

Table II. Details of the inclusion criteria

Age $\leq 40$ yrs at time of surgery

Surgery performed at Edinburgh Royal Infirmary

No associated injury or surgery to the posterior cruciate ligament

No revision of reconstruction of the $A C L$

Concise notes dictated at time of surgery

Table III. The incidence of meniscal tears in patients with reconstruction of the ACL carried out within 12 months of injury and, thereafter by number and percentage

\begin{tabular}{llr}
\hline & \multicolumn{2}{l}{ Time to surgery (mths) } \\
\cline { 2 - 4 } Type of meniscal tear & $\mathbf{0}$ to $\mathbf{1 2}$ & $\mathbf{>} \mathbf{1 2}$ \\
\hline None & $60(58.3)$ & $23(28.8)$ \\
Medial & $21(20.4)$ & $32(40.0)$ \\
Lateral & $18(17.5)$ & $16(20.0)$ \\
Both & $4(3.8)$ & $9(11.2)$ \\
\hline
\end{tabular}

men and 48 women with a mean age of 27 years (16 to 40 ). The age and gender distributions across the groups are shown in Table I. The mean time from injury to surgery was 27 months (1 week to 248 months; median 10 months). Reconstruction was carried out using a quadruple hamstring graft in 159 and a middle-third patellar tendon graft in 24 patients. The inclusion criteria are shown in Table II. All the surgical procedures were carried out by, or supervised by the senior author (JFK) who recorded the presence and type of meniscal tear and the type of degenerative change, if any, in the knee.

All the records were reviewed to evaluate the incidence of meniscal tears and degeneration at surgery. Additional details such as age, gender, the mechanism of injury, the time to surgery, the type of graft, the operative findings and associated injuries or surgery were noted. Meniscal surgery done before or after reconstruction of the ACL was also recorded. The operative findings included the location and type of meniscal tear and the degree of degenerative change, if any.

The incidence of meniscal tears and degenerative change was determined and then compared with the time to surgery. The relationships between meniscal tears and degenerative change to time to surgery were then analysed to determine whether a delay in surgery resulted in an increased incidence of meniscal tears and/or degenerative change.

The patients were divided into groups according to the time to surgery. The ACL reconstructions performed up to
12 months after injury were described as early and those after 12 months from injury as delayed reconstructions. The patients were then divided into groups of smaller time scales to examine the relationships more closely. These were: A) 0 to 12 months; B) 13 to 24 months; C) 25 to 36 months; D) 37 to 48 months and E) more than 48 months.

There were 103 patients in the early surgery group (0 to 12 months after injury) and 80 in the delayed surgery group (> 12 months) (Table I). As regards the groups with smaller time scales, there were 103 patients in group A, 22 in group B, 18 in group C, 13 in group D and 27 in group E.

Statistical analysis. Categorical data were analysed using the chi-squared test and non-parametric data were analysed using the Mann-Whitney U test. A p value of 0.05 was considered significant.

\section{Results}

Incidence of meniscal tears. The incidence of meniscal tears in the early and delayed groups is shown in Table III. Meniscal tears which occurred after injury to the ACL, but were dealt with at a previous arthroscopic procedure were included. There was a significant difference in the incidence of meniscal tears between patients treated in the early group and in those undergoing reconstruction of the ACL after 12 months (chi-squared test; $\mathrm{p}<0.001$ ). There were $21(20.4 \%)$ medial meniscal tears in the early group compared with $32(40 \%)$ in the delayed group. The incidence of lateral meniscal tears was similar in both groups, 18 $(17.5 \%)$ in the early group compared with $16(20 \%)$ in the delayed group. In the latter group there was also a higher incidence of patients with concomitant medial and lateral meniscal tears, four $(3.8 \%)$ in the early group compared with nine $(11.2 \%)$ in the late group.

The incidence of meniscal tears in the subgroups was then calculated (Table IV) and compared with the time to surgery. The lowest incidence of meniscal tears was in group $A$ and the highest in group D but differences between groups $\mathrm{B}$ to $\mathrm{E}$ were not significant. Medial meniscal tears were more common in groups B, C, and D. The number of patients with combined medial and lateral meniscal tears was highest in group $\mathrm{E}$.

Incidence of degenerative change. The incidence of degenerative change found at surgery was compared between the early and late groups, using the French Society of Arthroscopy (SFA) system (Table V). ${ }^{15}$ The incidence of grades 1,2 , 3 and 4 of degenerative change was higher in the delayed surgical group compared with the early group (MannWhitney $U$ test; $p<0.001)$. The incidence of grades 2,3 and 
Table IV. The incidence of meniscal tears in subgroups A to $\mathrm{E}$, by number and percentage

\begin{tabular}{llllll}
\hline \multirow{2}{*}{ Type of meniscal tear } & \multicolumn{4}{l}{ Time to surgery (mths) } \\
\cline { 2 - 6 } & $\mathbf{A}$ (0 to 12) & B (13 to 24) & C (25 to 36) & D (37 to 48) & E > 48 \\
\hline None & $60(58.3)$ & $7(31.8)$ & $5(27.8)$ & $3(23.1)$ & $8(29.6)$ \\
Medial & $21(20.4)$ & $9(40.9)$ & $9(50.0)$ & $6(46.2)$ & $8(29.6)$ \\
Lateral & $18(17.5)$ & $6(27.2)$ & $2(11.1)$ & $2(15.4)$ & $6(22.2)$ \\
Both & $4(3.8)$ & $0(0.0)$ & $2(11.1)$ & $2(15.4)$ & $5(18.5)$ \\
\hline
\end{tabular}

Table V. The incidence of degenerative change in early and delayed surgery, by number and percentage

\begin{tabular}{llr}
\hline \multirow{2}{*}{$\begin{array}{l}\text { French Society of Arthroscopy } \\
\text { grade of degenerative change }^{15}\end{array}$} & \multicolumn{2}{l}{ Time to surgery } \\
\cline { 2 - 3 } & $\mathbf{0}$ to $\mathbf{1 2}$ & $>\mathbf{1 2}$ \\
\hline 0 & $92(89.3)$ & $55(68.7)$ \\
1 & $4(3.9)$ & $7(8.7)$ \\
2 & $4(3.9)$ & $10(12.5)$ \\
3 & $1(0.97)$ & $3(3.7)$ \\
4 & $2(1.9)$ & $5(6.3)$ \\
\hline
\end{tabular}

4 of degenerative change was trebled in the delayed group compared with the early group. The incidence of grade 1 degenerative change was more than doubled in the delayed group.

Groups A to E were then analysed to assess the incidence of degenerative change found at surgery (Table VI). In the entire study group 147 patients had no evidence of degenerative change, and grade 4 degenerative change was seen only in seven patients. Two of these were in group A and five in group E. The incidence of degenerative change increased as time lengthened from injury, with patients in group $\mathrm{E}$ having the highest frequency of degenerative change.

The relationship of degenerative change to the timing of surgery after injury was not linear. Overall, the incidence in the early group was considerably less than that in the late group. However, in the subdivisions of the late group there was not a clear pattern of increasing degenerative change, although the highest incidence of degenerative change was found in group E, the group of patients in whom surgery had been carried out after 48 months.

\section{Discussion}

Our findings show a highly significant increase in meniscal tears and degeneration of the knee in patients undergoing reconstruction of the ACL more than 12 months after injury. The increase in meniscal tears in the late group was attributable principally to an increase in medial meniscal tears. The incidence of lateral meniscal tears remained relatively unchanged with time. This may indicate that lateral meniscal tears occur at the time of injury to the ACL or very soon after injury, whereas medial meniscal tears are acquired after the knee has been ACL-deficient for more than 12 months. The increase in meniscal tears and degenerative change after 12 months possibly coincides with a return to pre-injury levels of sporting activity. The data are consistent with the development of instability of the knee associated with a return to sport with increased torsional and shear forces resulting in acquired medial meniscal injuries and acceleration of degenerative change in the knee.

It has been demonstrated that the incidence of degenerative change increases when surgery is performed after 12 months from injury, even although most patients in both groups had no degenerative change at all. Across the subgroups B to E there was no clear pattern, although the most severe degrees of degenerative change were observed in group E, with the longest interval to surgery. The range of timing to operation in group $\mathrm{E}$ was very wide, from 52 to 248 months. Some degenerative change in a proportion of these knees would therefore be expected since ACL deficiency is well documented to be associated with the development of late degenerative change. ${ }^{1,3}$ Our findings indicate that reconstruction carried out within one year of injury was associated with a very low incidence of degenerative change. It remains to be seen whether a reconstruction carried out at this stage confers a longer-term benefit in the prevention of late degenerative change. Although satisfactory results can be achieved even with late reconstruction, ${ }^{10}$ the results in a degenerative knee have been shown not to match those of early reconstruction in a knee with normal articular cartilage. ${ }^{9}$

Only one other study has specifically evaluated the incidence of meniscal tears and osteochondral damage in acute

Table VI. The incidence of degenerative change at surgery in subgroups $A$ to $E$, by number and percentage

\begin{tabular}{llllll}
\hline \multirow{2}{*}{$\begin{array}{l}\text { French Society of Arthroscopy } \\
\text { grade of degenerative change }^{\mathbf{1 5}}\end{array}$} & \multicolumn{4}{l}{ Time to surgery (mths) } \\
\cline { 2 - 6 } & $\mathbf{A}$ (0 to 12) & $\mathbf{B}(\mathbf{1 3}$ to $\mathbf{2 4 )}$ & $\mathbf{C}(\mathbf{2 5}$ to $\mathbf{3 6})$ & $\mathbf{D}$ (37 to 48) & $\mathbf{E}>\mathbf{4 8}$ \\
\hline 0 & $92(89.3)$ & $15(68.1)$ & $17(94.4)$ & $10(76.9)$ & $13(48.1)$ \\
1 & $4(3.9)$ & $2(9.1)$ & $0(0.0)$ & $1(7.7)$ & $4(14.8)$ \\
2 & $4(3.9)$ & $3(13.6)$ & $1(5.6)$ & $2(15.4)$ & $1(3.7)$ \\
3 & $1(0.97)$ & $2(9.1)$ & $0(0.0)$ & $0(0.0)$ & $1(3.7)$ \\
4 & $2(1.9)$ & $0(0.0)$ & $0(0.0)$ & $0(0.0)$ & $5(18.5)$ \\
\hline
\end{tabular}


and chronic ACL-deficient knees. ${ }^{16}$ In that study, MRI findings were compared in the two groups. As in our study, there was a higher incidence of medial meniscal tears in chronic ACL-deficient knees, $78 \%$ in chronic ACL tears vs $40 \%$ in acute ACL tears. The study did not evaluate the effect of time from injury to reconstruction on the incidence of meniscal tears, nor was there a detailed assessment of degenerative change. Other studies have looked generally at the incidence of meniscal tears and degenerative change in the ACL-deficient knee ${ }^{1-5}$ and also at the incidence of meniscal pathology in adolescent patients with tears of the ACL. ${ }^{12}$ Not surprisingly, these studies indicated that the incidence of degenerative change and meniscal tears increased in the chronic ACL-deficient knee. No previous study has attempted to relate the development of this pathology to the timing of reconstruction of the ACL. In a previous study we have shown there is no advantage in terms of graft failure or functional outcome of immediate versus delayed reconstruction of the ACL. ${ }^{14}$ In that study, however, the delayed group was scheduled to undergo reconstruction after a period of rehabilitation lasting from 8 to 12 weeks, and therefore delay to reconstruction was minimal.

The main strength of our study was the large number of patients with prospective documentation of arthroscopic findings. All operative findings were documented by the same surgeon (JFK) who also performed or supervised every operation. This minimised the observer variability in the classification of the type of meniscal tear and the degree of degenerative change. No previous study has evaluated the incidence of meniscal tears and degenerative change and related this to the timing of surgery. We excluded patients older than 40 years of age since a proportion of these patients could be expected to have developed degenerative change independent of a tear in the ACL. Complex multiple ligament reconstructions were also excluded since these have been documented to have a high incidence of degenerative change. ${ }^{17}$ The age and gender distribution across the groups was comparable. The SFA classification score ${ }^{15}$ was used to categorise the degeneration found in the knee, thereby allowing an objective documentation of the findings to be made.

The main weakness of our study was the small number of patients in the subdivisions of the late group. The differences between these subgroups may have been significant, but our numbers were not sufficient to be certain of this. A randomised study comparing early and late reconstruction would be ideal but there are practical and ethical difficulties in conducting such a trial in modern orthopaedic practice. In particular, it would be difficult to persuade patients to consent to a delay in treatment with a symptomatic un- stable knee and run the risk of developing meniscal tears and degenerative change.

The relationship of reconstruction of the ACL to subsequent arthritis remains controversial, but it does appear to reduce the risk of development of meniscal tears. ${ }^{1}$ There is evidence from a previous evaluation of outcome after reconstruction of the ACL that early reconstruction is associated with better results than late reconstruction, often carried out in the presence of meniscal tears or degenerative change. ${ }^{1}$

To verify the findings of our study a larger prospective study would be of value. A functional follow-up could be incorporated into the design of the study. ${ }^{18}$ This would show whether the presence of meniscal tears and degenerative change compromised the outcome of surgery.

No benefits in any form have been received or will be received from a commercial party related directly or indirectly to the subject of this article.

\section{References}

1. Fithian DC, Paxton LW, Goltz DH. Fate of the anterior cruciate ligament-injured knee. Orthop Clin North Am 2002;33:621-36.

2. Steenbrugge F, Verdonk R, Verstraete K. Long-term assessment of arthroscopic meniscus repair: a 13-year follow-up study. Knee 2002;9:181-7.

3. Ruiz AL, Kelly M, Nutton RW. Arthroscopic ACL reconstruction: a 5-9 year followup. Knee 2002;9:197-200.

4. Jarvela $\mathbf{T}$, Kannus $\mathbf{P}$, Jarvinen $\mathbf{M}$. Anterior cruciate ligament reconstruction in patients with or without accompanying injuries: a re-examination 5-9 years after reconstruction. Arthroscopy 2001;17:818-25

5. Chol C, Ait S, Selmi T, et al. Seventeen year outcome after anterior cruciate ligament reconstruction with an intact or repaired medial meniscus. Rev Chir Orthop Reparatrice Appar Mot 2002;88:157-62 (in French).

6. Orfaly RM, McConkey JP, Regan WD. The fate of meniscal tears after anterior cruciate ligament reconstruction. Clin J Sport Med 1998:8:102-5.

7. Barber FA, Click SD. Meniscus repair rehabilitation with concurrent anterior cruciate reconstruction. Arthroscopy 1997;13:433-7.

8. Seitz H, Marlovitz S, Wielke T, Vescei V. Meniscus lesions after isolated anterior cruciate ligament rupture. Wein Klin Wochenschr 1996;108:727-30 (in German).

9. Thompson W0, Fu FH. The meniscus in the cruciate-deficient knee. Clin Sports Med 1993:12:771-96.

10. Richter M, Bosch U, Wippermann B, Hofmann A, Krettek C. Comparison of surgical repair or reconstruction of the cruciate ligaments versus non-surgical treatments in patients with traumatic knee dislocations. Am J Sports Med 2002;30:718-27.

11. Wittenberg RH, Oxfort HU, Plafki C. A comparison of conservative and delayed surgical treatment of anterior cruciate ligament ruptures: a matched pair analysis. Int Orthop 1998;22:145-8.

12. Millett PJ, Willis AA, Warren RF. Associated injuries in pediatric and adolescent anterior cruciate tears: does a delay in treatment increase the risk of meniscal tear? Arthroscopy 2002;18:955-9.

13. Cipolla M, Scala A, Gianni E, Puddu G. Different patterns of meniscal tears in acute anterior cruciate ligament (ACL) ruptures and in chronic ACL-deficient knees: classification, staging and timing of treatment. Knee Surg Sports Traumatol Arthrosc 1995:3:130-4.

14. Meighan A, Keating JF, Will E. Early versus delayed reconstruction for acute ACL tears: a prospective randomised trial. J Bone Joint Surg [Br] 2003:85-B:521-4.

15. Dougados M, Ayral X, Listrat V, et al. The SFA system for assessing articular cartilage lesions at arthroscopy of the knee. Arthroscopy 1994;10:69-77.

16. Dimond PM, Fadale PD, Hulstyn MJ, Tung GA, Greisberg J. A comparison of MRI findings with acute and chronic ACL tears. Am J Knee Surg 1998;11:153-9.

17. Werier J, Keating JF, Meek RN. Complete dislocation of the knee: the long term results of ligamentous reconstruction. Knee 1998:5:255-60.

18. Tegner Y, Lysholm J. Rating systems in the evaluation of knee ligament injuries. Clin Orthop 1985;198:43-9. 